ISSN 1980-5098

\title{
AVALIAÇÃO DA REGENERAÇÃO NATURAL EM ÁREA DE RESTAURAÇÃO ECOLÓGICA E MATA CILIAR DE REFERÊNCIA
}

\section{EVALUATION OF THE NATURAL REGENERATION IN A RESTORATION PLANTING AREA AND IN A REFERENCE RIPARIAN FOREST}

\author{
Debora Aline da Fonseca ${ }^{1}$ Amanda Ratier Backes ${ }^{2}$ Milena Fermina Rosenfield ${ }^{3}$ \\ Gerhard Ernst Overbeck ${ }^{4}$ Sandra Cristina Müller ${ }^{5}$
}

\begin{abstract}
RESUMO
Áreas ribeirinhas são foco recorrente de restauração ecológica, devido a sua importância para a manutenção de serviços ecossistêmicos. Entretanto, poucos estudos têm de fato avaliado o sucesso de intervenções ativas em restaurar funções ou processos ecossistêmicos. A regeneração natural é um processo sucessional, cuja avaliação pode indicar o estado e o potencial de resiliência do ecossistema em áreas sob restauração. O presente estudo comparou padrões de regeneração natural de uma área de restauração ecológica (com plantio de mudas nativas há 10 anos) com a mata ciliar de referência, em Cachoeirinha, Rio Grande do Sul, Brasil. Para tanto, foi realizado o levantamento das espécies arbóreas e arbustivas presentes no estrato superior $\left(\mathrm{DAP} \geq 5 \mathrm{~cm}\right.$ ) e inferior (altura $>30 \mathrm{~cm}$ e DAP $<5 \mathrm{~cm}$ ), em 40 parcelas de $100 \mathrm{~m}^{2}$ cada, considerando o plantio (restauração) e a mata ciliar remanescente (referência). Cada estrato e tratamento (referência vs. restauração) foi avaliado quanto aos descritores fitossociológicos, padrões de estrutura e composição, riqueza e similaridade entre as comunidades. Os resultados demonstraram que a área de restauração apresenta composição de espécies e estrutura diferenciada com relação à referência, especialmente para o estrato superior. No estrato inferior (regeneração natural), o número de indivíduos, a altura média e a riqueza de espécies já não diferiram da referência. A composição de espécies em regeneração ainda foi distinta, porém, esta foi mais similar entre si do que as demais comparações entre estratos, indicando que espécies não plantadas foram capazes de se estabelecer nas áreas de restauração.
\end{abstract}

Palavras-chave: riqueza; composição; estrutura; sucessão.

\section{ABSTRACT}

Riparian areas are a recurrent focus of ecological restoration due to their importance for the maintenance of ecosystem services. However, few studies have evaluated the success of active interventions in restoring ecosystem functions and processes. The natural regeneration is a successional process and its evaluation might reveal the state and the potential of the ecosystem resilience in forest areas undergoing restoration.

1 Bióloga, Mestranda em Agronomia pelo Programa de Pós-Graduação em Ecologia, Instituto de Biociências, Universidade Federal do Rio Grande do Sul. Av. Bento Gonçalves, 9500, Bairro Agronomia, CEP 91501-970, Porto Alegre (RS), Brasil. deboraafonseca@yahoo.com.br

2 Bióloga, Mestranda em Ecologia Aplicada pela Universidade de Poitiers, França, Instituto de Biociências, Universidade Federal do Rio Grande do Sul. Av. Bento Gonçalves, 9500, Bairro Agronomia, CEP 91501-970, Porto Alegre (RS), Brasil. amandaratier@gmail.com

3 Bióloga, Doutoranda em Ecologia, Instituto de Biociências, Universidade Federal do Rio Grande do Sul. Av. Bento Gonçalves, 9500, Bairro Agronomia, CEP 91501-970, Porto Alegre (RS), Brasil. milenarosenfield@gmail.com

4 Engenheiro Ambiental, Dr., Professor do Departamento de Botânica, Instituto de Biociências, Universidade Federal do Rio Grande do Sul. Av. Bento Gonçalves, 9500, Bairro Agronomia, CEP 91501-970, Porto Alegre (RS), Brasil. gerhard.overbeck@ufrgs.br

5 Bióloga, Dr ${ }^{\mathrm{a}}$., Professora do Departamento de Ecologia, Instituto de Biociências, Universidade Federal do Rio Grande do Sul. Av. Bento Gonçalves, 9500, Bairro Agronomia, CEP 91501-970, Porto Alegre (RS), Brasil. sandra. muller@ufrgs.br

Recebido para publicação em 30/10/2014 e aceito em 9/09/2015 
The present study aimed to compare natural regeneration of a riparian area that is undergoing restoration (planting of native trees, 10 years ago) with a reference forest area, in Cachoeirinha, Rio Grande do Sul state, Brazil. We conducted a survey of trees and shrubs in the upper stratum (DBH $\geq 5 \mathrm{~cm})$ and the lower stratum $(>30 \mathrm{~cm}$ in height and DBH $>5 \mathrm{~cm})$ in a total of 40 plots $\left(100 \mathrm{~m}^{2}\right.$ each), within the planting (restoration) and the remnant forest (reference). For each stratum and treatment (reference vs. restoration) we analyzed phytosociological parameters, patterns of structure and composition, and similarity among plots. The results showed significant differences in relation to structure and species composition, especially for the upper stratum. For the lower stratum (natural regeneration), abundance, height, and species richness were similar between the restoration and the reference areas. Species composition in regeneration remained distinct. However, its similarity value was higher than any other comparison among strata, indicating that species that were not planted were able to establish in the planted areas.

Keywords: richness; composition; structure; succession.

\section{INTRODUÇÃO}

A restauração ecológica utiliza práticas que visam assistir à recuperação de ecossistemas degradados, resgatando aspectos estruturais e funcionais observados nos sistemas de referência, a fim de reestabelecer processos ecológicos associados à estabilidade, sucessão e resiliência do sistema (SOCIETY FOR ECOLOGICAL RESTORATION, 2004; TRES et al., 2007; NERY et al., 2013). Isto é almejado, por exemplo, para áreas degradadas ribeirinhas, as quais são de extrema importância para a manutenção de serviços ecossistêmicos e fluxos ecológicos (RODRIGUES; LEITÃO FILHO, 2001). A meta de restabelecer processos ecológicos, como ciclos hidrológicos, produtividade e interações bióticas, deve ser preferencialmente almejada e instituída em projetos de restauração, em vez de simplesmente avaliar o retorno de uma estrutura e composição particular (STANTURF et al., 2014). Por outro lado, são poucos os estudos que de fato têm avaliado métricas ou variáveis associadas a tais processos (WORTLEY et al., 2013).

As técnicas de restauração de ecossistemas degradados geralmente incluem a eliminação da fonte de distúrbios, intervenções no solo, eliminação ou manejo de espécies invasoras e introdução de espécies desejadas. No Brasil, a maioria dos trabalhos de restauração ecológica se restringe a ecossistemas florestais, embora também existam muitas áreas degradadas em ecossistemas campestres e poucos estudos de restauração (OVERBECK et al. 2013). Em ecossistemas florestais, a introdução de espécies é geralmente feita através do plantio de mudas, nos quais um dos objetivos é facilitar a chegada (atração de dispersores) e o estabelecimento de espécies lenhosas a partir da melhoria das condições de sombreamento e temperatura nestes locais (SOCIETY FOR ECOLOGICAL RESTORATION, 2004; NERY et al., 2013; HOLL, 2013).

A influência de plantios sobre a regeneração natural em áreas de restauração florestal tem sido observada (SUGANUMA et al., 2014), bem como há resultados que ressaltam a importância de fatores como disponibilidade de propágulos, características da paisagem do entorno e presença de gramíneas invasoras (KAUANO et al., 2013; PEREIRA et al., 2013). Neste sentido, a avaliação da regeneração natural através de dados sobre estrutura, composição e diversidade de espécies lenhosas aparece como uma ferramenta amplamente utilizada e com potencial de indicar processos ecológicos associados à dinâmica sucessional das comunidades em restauração (MELO; DURIGAN, 2007; RIGUEIRA; MARIANO-NETO, 2013; PIOVESAN et al., 2013). Análises de trajetória das comunidades em regeneração, quando comparadas com padrões de áreas de referência, permitem inferir sobre barreiras ecológicas na regeneração natural de determinadas espécies ou grupos funcionais (GUARIGUATA; OSTERTAG, 2001; SUGANUMA et al., 2014). Assim, entende-se que avaliar a regeneração natural como um processo de retomada da sucessão possibilita estabelecer relações entre padrões de composição e diversidade de espécies observados e mecanismos que aceleram ou retardam este processo.

O objetivo geral deste trabalho foi avaliar padrões de composição, estrutura e riqueza de espécies de árvores e arbustos na regeneração natural em área de restauração de floresta ciliar em relação à referência (remanescentes). A restauração foi implementada em áreas adjacentes a remanescentes estreitos de floresta ciliar, com plantio de mudas de espécies nativas. Os objetivos específicos foram (i) verificar a composição de espécies e os padrões de distribuição e abundância destas nas áreas em restauração (plantio) e referência; 
(ii) avaliar a similaridade florística entre os estratos superior e inferior, bem como a estrutura (número de indivíduos, área basal, altura) e a riqueza de espécies das áreas de plantio e referência, e (iii) avaliar a influência da riqueza de espécies de mudas plantadas sobre a riqueza do estrato inferior das áreas em restauração. Considerando o potencial efeito favorável do plantio de mudas sobre a chegada e estabelecimento de espécies florestais (CARNEVALE; MONTAGNINI, 2002) espera-se que a similaridade entre as áreas seja maior para o estrato inferior quando comparado ao estrato superior, e que haja relações positivas entre a riqueza de espécies do estrato inferior, a densidade de indivíduos e o número de espécies plantadas presentes no estrato superior das áreas de plantio. Ou seja, que plantios mais homogêneos (com baixa riqueza de espécies) e com menor proporção de sombreamento e poleiros (menor densidade de indivíduos adultos) resultem em menor riqueza de espécies no estrato regenerante.

\section{MATERIAL E MÉTODOS}

\section{Área de estudo}

O estudo foi realizado em uma formação ribeirinha situada em uma paisagem de mosaicos campofloresta no Parque Ambiental Souza Cruz, pertencente à Empresa Souza Cruz, no município de Cachoeirinha, Rio Grande do Sul, Brasil. As coordenadas centrais do Parque são $29^{\circ} 52^{\prime} 41,53^{\prime \prime}$ S e $51^{\circ} 05^{\prime} 50,22^{\prime \prime}$ W, com altitude em torno dos $30 \mathrm{~m}$. O clima, segundo o sistema de Köppen, é do tipo Cfa, que se caracteriza por apresentar chuvas durante todos os meses do ano e possuir a temperatura do mês mais quente superior a $22^{\circ} \mathrm{C}$, e a do mês mais frio superior a $3^{\circ} \mathrm{C}$, com temperatura média anual de $19,7^{\circ} \mathrm{C}$ e precipitação média anual de $1.538 \mathrm{~mm}$ (MORENO, 1961). O Parque localiza-se na Unidade Geomorfológica Planície Lagunar e o solo é caracterizado como Podzólico Vermelho-Amarelo com textura arenosa/argilosa (IBGE, 2002).

A área de estudo está localizada às margens do arroio Nazário, um afluente da margem esquerda do arroio Sapucaia, inserido na bacia hidrográfica do Rio dos Sinos (TEIXEIRA, 2007). É uma região de transição entre o bioma Mata Atlântica e o bioma Pampa, segundo a definição dos biomas brasileiros do IBGE (2004). A vegetação ciliar da área de estudo se enquadra na classificação de Floresta Estacional Semidecidual (TEIXEIRA, 2007). De acordo com Leite (2002), a Floresta Estacional Semidecidual desta região, por estar em uma região de transição entre a região costeira e a Floresta Estacional do interior, sofre influência oceânica na seleção florística. O estrato superior é formado por espécies provenientes tanto da Floresta Estacional Decidual quanto da Floresta Ombrófila Densa, possuindo uma fisionomia marcada pela ocorrência de espécies sempre-verdes da zona costeira.

Segundo informações locais, a área do Parque Ambiental Souza Cruz já foi utilizada para criação de gado e apresenta remanescentes de campo nativo. Hoje, na ausência de pastejo, roçadas ou fogo, a fisionomia das áreas de campo encontra-se caracterizada pela presença de arbustos (vassouras) e pela expansão de árvores tipicamente florestais, com locais de maior e menor densidade de lenhosas entremeados por áreas mais abertas (predomínio de gramíneas). A mata ciliar remanescente junto à margem do arroio é bastante estreita (cerca de 10 a $15 \mathrm{~m}$ ) e encontra-se em processo de regeneração natural desde que foi implementado o parque, no ano de 2004. Há 10 anos, foi realizado um plantio de mudas de espécies nativas da região, entre elas, aroeira-vermelha (Schinus terebinthifolius Raddi), tarumã (Vitex megapotamica (Spreng.) Moldenke), araçá (Psidium cattleyanum Sabine) e açoita-cavalo (Luehea divaricata (Mart.)). O plantio, realizado com espaçamento de aproximadamente $2 \times 2 \mathrm{~m}$ entre mudas e sem arranjo específico das espécies, objetivou ampliar a faixa de margem ciliar, buscando alcançar $30 \mathrm{~m}$ de vegetação florestal contínua nos dois lados do curso d'água, conforme determinava a legislação da época.

\section{Levantamento de dados}

Na primavera de 2013, foi realizado levantamento das espécies lenhosas do estrato superior (diâmetro a altura do peito (DAP) $\geq 5 \mathrm{~cm}$ ) e do estrato inferior (altura $>30 \mathrm{~cm}$ e DAP $<5 \mathrm{~cm}$ ). Foram considerados dois tratamentos distintos: áreas com plantio de mudas (área de restauração) e áreas de floresta ciliar remanescente (área de referência). Em cada tratamento, foram instaladas de forma sistemática e pareada 20 parcelas (unidades amostrais - UA) retangulares de 5 x $20 \mathrm{~m}\left(100 \mathrm{~m}^{2}\right)$, sendo a maior extensão da UA 
paralela ao curso d'água. Nestas UA foram medidos e identificados todos os indivíduos do estrato superior, sendo distinguidos aqueles que haviam sido plantados na área de restauração. Para o estrato inferior, a amostragem foi realizada em cinco subparcelas de $2 \times 2 \mathrm{~m}$, inseridas sistematicamente dentro de cada UA do estrato superior. As espécies foram identificadas em campo e com auxílio de chaves taxonômicas (SOBRAL et al., 2006) ou ainda por consulta a especialistas e ao Herbário do Departamento de Botânica da Universidade Federal do Rio Grande do Sul (ICN). A nomenclatura utilizada para agregação em famílias seguiu a proposta do APG III (ANGIOSPERM PHYLOGENY GROUP, 2009).

\section{Análise dos dados}

A composição de espécies em cada tratamento foi inicialmente avaliada a partir do valor de importância de cada espécie, calculado a partir dos descritores fitossociológicos clássicos (frequência relativa, densidade relativa e dominância relativa) (MORO; MARTINS, 2011). Em seguida, o grau de semelhança entre os estratos superior e inferior, e entre os tratamentos, foi avaliado através do coeficiente de similaridade de Jaccard, considerando a área amostral total por tratamento.

As diferenças na riqueza de espécies e estrutura das comunidades entre os tratamentos foram avaliadas por ANOVA via teste de aleatorização e 10.000 iterações, considerando os pares de parcela como blocos. Como estrutura, foram consideradas as seguintes variáveis: número de indivíduos, altura média, variância da altura, altura máxima e área basal total, sendo este último avaliado apenas para o estrato superior.

Para verificar os padrões da composição de espécies dos estratos superior e inferior, dada a distribuição e abundância das mesmas nos tratamentos (plantio vs. referência), foram realizadas análises de ordenação e as hipóteses de diferenças na composição foram testadas através de MANOVA (PILLAR, 2013). Primeiramente, foi feita uma análise de ordenação por coordenadas principais (PCoA) para o conjunto total de dados (os dois estratos de ambos tratamentos), considerando a presença/ausência das espécies e a similaridade de Jaccard entre UA, para avaliar exclusivamente a composição de espécies. Depois, foi avaliado o padrão por estrato (superior e inferior) com análises de ordenação aplicadas aos dados de densidade por espécie como descritor das UA, a fim de avaliar o padrão de composição dada a distribuição e abundância das espécies nas áreas. Neste caso, as PCoA foram realizadas com base na medida de distância de Corda entre UA, a qual considera distâncias euclidianas sobre dados previamente normalizados dentro de unidades (LEGENDRE; LEGENDRE, 2012). Assim, esta medida possibilita observar as distâncias entre UA e mantém as relações de abundância entre as espécies dentro de cada UA. Estas distâncias, por sua vez, são mantidas tanto quanto possível no método de coordenadas principais, sem ajustes ou qualquer rotação das unidades no espaço multidimensional, sendo a variação dos dados maximizada nos primeiros eixos da ordenação (LEGENDRE; LEGENDRE 2012). As MANOVA foram realizadas com base nas respectivas matrizes de distância (ora Jaccard, ora distância de Corda), via testes de aleatorização com 1.000 iterações.

Para verificar as relações entre a riqueza de espécies regenerantes e a riqueza de espécies plantadas ou o número de indivíduos plantados, foram ajustados modelos de regressão linear simples através de teste de aleatorização, considerando seus pressupostos, com 5.000 interações (MANLY, 1991). As análises foram realizadas com o software MULTIV (por V.D. Pillar, disponível em http://ecoqua.ecologia.ufrgs.br/ software).

\section{RESULTADOS}

\section{Composição florística}

Ao todo foram amostradas 96 espécies, distribuídas em 37 famílias. A família com maior riqueza foi Myrtaceae, com 14 espécies, seguida de Fabaceae com seis e Lauraceae com cinco espécies (Tabela 1). No estrato superior da mata ciliar remanescente foram amostrados 450 indivíduos vivos (1.125 ind.ha-1), mais 38 mortos ainda em pé, distribuídos em 51 espécies e 26 famílias, sendo Myrtaceae a família com maior riqueza de espécies (oito) e Sebastiania serrata (Baill. ex Müll. Arg.) Müll. Arg. a espécie com maior valor de importância (29,8\%). O estrato superior da área em restauração apresentou 232 indivíduos vivos 
TABELA 1: Valores de densidade de espécies nos estratos superior $\left(4.000 \mathrm{~m}^{2}\right)$ e inferior $\left(800 \mathrm{~m}^{2}\right)$, considerando a área de referência (floresta ciliar) e a área de restauração (plantio), em Cachoeirinha, RS. * = espécies utilizadas no plantio.

TABLE 1: Values of stem density among species in the upper $\left(4.000 \mathrm{~m}^{2}\right)$ and lower strata $\left(800 \mathrm{~m}^{2}\right)$, regarding sites of reference (riparian forest) and restoration sites (planting), in Cachoeirinha, RS state. ${ }^{*}=$ species used in the planting.

\begin{tabular}{|c|c|c|c|c|c|c|}
\hline \multirow{2}{*}{ Família } & \multirow{2}{*}{ Espécie } & \multirow{2}{*}{ Rótulo- } & \multicolumn{2}{|c|}{ Estrato superior } & \multicolumn{2}{|c|}{ Estrato inferior } \\
\hline & & & Referência & Plantio & Referência & Plantio \\
\hline Acanthaceae & Justicia brasiliana Roth & Jubr & 0 & 0 & 15 & 0 \\
\hline \multirow{2}{*}{ Anacardiaceae } & Lithraea brasiliensis Marchand & Libr & 4 & 0 & 0 & 1 \\
\hline & Schinus terebinthifolius Raddi $^{1}$ & Scte & 1 & 60 & 0 & 52 \\
\hline \multirow{2}{*}{ Annonaceae } & Annona sp. ${ }^{1}$ & Ansp & 0 & 2 & 0 & 2 \\
\hline & Annona sylvatica A. St.-Hil. & Ansy & 1 & 0 & 0 & 0 \\
\hline Apocynaceae & Aspidosperma australe Müll. Arg. ${ }^{1}$ & Asau & 0 & 1 & 0 & 1 \\
\hline Aquifoliaceae & Ilex dumosa Reissek & Ildu & 0 & 0 & 0 & 1 \\
\hline Arecaceae & Syagrus romanzoffiana (Cham.) Glassman & Syro & 2 & 0 & 30 & 18 \\
\hline \multirow{3}{*}{ Asteraceae } & Gochnatia polymorpha (Less.) Cabrera & Gopo & 0 & 0 & 0 & 2 \\
\hline & Vernonanthura tweedieana (Baker) H. Rob. & Vetw & 0 & 0 & 0 & 2 \\
\hline & Handroanthus heptaphyllus (Vell.) Mattos & Hahe & 0 & 0 & 3 & 0 \\
\hline \multirow[t]{2}{*}{ Bignoniaceae } & Handroanthus umbellatus (Sond.) Mattos & Haum & 2 & 0 & 0 & 0 \\
\hline & Jacaranda micranta Cham. $^{1}$ & Jami & 0 & 3 & 0 & 0 \\
\hline Boraginaceae & Cordia americana (L.) Gottschling \& J.S. Mill. ${ }^{1}$ & Coam & 3 & 1 & 2 & 0 \\
\hline Cactaceae & Cereus hildmannianus K. Schum. & Cehi & 1 & 0 & 0 & 0 \\
\hline \multirow{2}{*}{ Cannabaceae } & Celtis iguanaea (Jacq.) Sarg. ${ }^{1}$ & Ceig & 1 & 2 & 6 & 5 \\
\hline & Trema micranta (L.) Blume & Trmi & 1 & 0 & 0 & 0 \\
\hline Celastraceae & Maytenus dasyclados Mart. & Mada & 1 & 0 & 19 & 2 \\
\hline \multirow{3}{*}{ Erythroxylaceae } & Erythroxylum argentinum O.E. Schulz ${ }^{1}$ & Erar & 6 & 1 & 5 & 13 \\
\hline & Erythroxylum deciduum A. St.-Hil. ${ }^{1}$ & Erde & 3 & 15 & 0 & 4 \\
\hline & Sapium glandulosum (L.) Morong & Sagl & 1 & 5 & 1 & 14 \\
\hline \multirow{4}{*}{ Euphorbiaceae } & Sebastiania brasiliensis Spreng. & Sebr & 4 & 0 & 3 & 0 \\
\hline & Sebastiania serrata (Baill. ex Müll. Arg.) Müll. Arg. & Sese & 208 & 1 & 140 & 23 \\
\hline & Apuleia leiocarpa (Vogel) J.F. Macbr. ${ }^{1}$ & Aple & 0 & 8 & 7 & 1 \\
\hline & Inga marginata Willd. ${ }^{1}$ & Inma & 0 & 13 & 0 & 15 \\
\hline \multirow{4}{*}{ Fabaceae } & Inga vera Willd. ${ }^{1}$ & Inve & 0 & 7 & 0 & 13 \\
\hline & Mimosa bimucronata (DC.) Kuntze ${ }^{1}$ & Mibi & 8 & 10 & 0 & 48 \\
\hline & Parapiptadenia rigida (Benth.) Brenan ${ }^{1}$ & Pari & 1 & 6 & 1 & 5 \\
\hline & Peltophorum dubium (Spreng.) Taub. & Pedu & 2 & 1 & 0 & 0 \\
\hline \multirow{4}{*}{ Lamiaceae } & Aegiphila integrifolia (Jacq.) B.D. Jacks. & Aein & 5 & 0 & 2 & 2 \\
\hline & Vitex megapotamica (Spreng.) Moldenke ${ }^{1}$ & Vime & 3 & 7 & 0 & 1 \\
\hline & Cinnamomum verum J. Presl & Cive & 0 & 0 & 0 & 3 \\
\hline & Endlicheria paniculata (Spreng.) J.F. Macbr. & Enpa & 0 & 0 & 7 & 1 \\
\hline \multirow[t]{3}{*}{ Lauraceae } & Nectandra grandiflora Nees \& Mart. ex Nees & Negr & 6 & 0 & 133 & 61 \\
\hline & Ocotea puberula (Rich.) Nees ${ }^{1}$ & Ocpu & 9 & 2 & 1 & 6 \\
\hline & Ocotea pulchella (Nees \& Mart.) Mez & Ocpul & 2 & 0 & 9 & 1 \\
\hline
\end{tabular}


TABELA 1: Continuação...

TABLE 1: Continued...

\begin{tabular}{|c|c|c|c|c|c|c|}
\hline \multirow{2}{*}{ Família } & \multirow{2}{*}{ Espécie } & \multirow{2}{*}{ Rótulo- } & \multicolumn{2}{|c|}{ Estrato superior } & \multicolumn{2}{|c|}{ Estrato inferior } \\
\hline & & & Referência & Plantio & Referência & Plantio \\
\hline \multirow[t]{2}{*}{ Loganiaceae } & Strychnos brasiliensis (Spreng.) Mart. & Stbr & 0 & 0 & 9 & 5 \\
\hline & Luehea divaricata Mart. $^{1}$ & Ludi & 16 & 8 & 2 & 41 \\
\hline \multirow{4}{*}{ Malvaceae } & Indeterminada 1 & & 0 & 0 & 1 & 11 \\
\hline & Indeterminada 2 & & 0 & 0 & 0 & 2 \\
\hline & Triumfetta semitriloba Jacq. & Trse & 0 & 0 & 30 & 11 \\
\hline & Leandra australis (Cham.) Cogn. & Leau & 0 & 0 & 4 & 195 \\
\hline \multirow{3}{*}{ Melastomataceae } & Miconia cinerascens Miq. & Mici & 0 & 0 & 4 & 18 \\
\hline & Miconia sellowiana Naudin & Mise & 2 & 0 & 25 & 10 \\
\hline & Cedrela fissilis Vell. & Cefi & 3 & 0 & 0 & 1 \\
\hline \multirow[t]{2}{*}{ Meliaceae } & Guarea macrophylla Vahl & Guma & 0 & 0 & 1 & 1 \\
\hline & Trichilia elegans A. Juss. & Trel & 0 & 0 & 2 & 2 \\
\hline Monimiaceae & Mollinedia elegans Tul. & Moel & 1 & 0 & 320 & 19 \\
\hline \multirow{8}{*}{ Moraceae } & Ficus luschnathiana (Miq.) Miq. & Filu & 2 & 0 & 2 & 0 \\
\hline & $\begin{array}{l}\text { Sorocea bonplandii (Baill.) W.C. Burger, Lanj. \& Wess. } \\
\text { Boer }\end{array}$ & Sobo & 1 & 0 & 42 & 0 \\
\hline & Blepharocalyx salicifolius (Kunth) O. Berg & Blsa & 4 & 0 & 3 & 13 \\
\hline & Calyptranthes concinna DC. & Caco & 0 & 0 & 10 & 3 \\
\hline & Campomanesia rhombea $\mathrm{O}$. Berg. & Carh & 1 & 0 & 16 & 6 \\
\hline & Eugenia hiemalis Cambess. & Euhi & 4 & 0 & 112 & 18 \\
\hline & Eugenia pyriformis Cambess. & Eupy & 0 & 0 & 0 & 1 \\
\hline & Eugenia uniflora L. $^{1}$ & Euun & 0 & 2 & 5 & 41 \\
\hline \multirow{8}{*}{ Myrtaceae } & Eugenia verticillata (Velloso) Angely & Euve & 2 & 0 & 26 & 0 \\
\hline & Myrcia glabra (O. Berg) D. Legrand & Mygl & 5 & 0 & 63 & 26 \\
\hline & Myrcia multiflora (Lam.) DC. & Mymu & 2 & 0 & 57 & 50 \\
\hline & Myrcia oblongata DC. & Myob & 0 & 0 & 0 & 68 \\
\hline & Myrcia palustres DC. & Mypa & 3 & 0 & 7 & 5 \\
\hline & Myrcianthes gigantea (D. Legrand) D. Legrand & Mygi & 3 & 0 & 8 & 2 \\
\hline & Myrcianthes pungens (O. Berg) D. Legrand & Mypu & 0 & 0 & 0 & 8 \\
\hline & Psidium cattleyanum Sabine ${ }^{1}$ & Psca & 0 & 56 & 4 & 52 \\
\hline \multirow[t]{2}{*}{ Piperaceae } & Piper aduncum L. & Piad & 0 & 0 & 176 & 0 \\
\hline & Myrsine coriacea (Sw.) R. Br. ex Roem. \& Schult. & Myco & 7 & 6 & 18 & 283 \\
\hline \multirow[t]{2}{*}{ Primulaceae } & Myrsine lorentziana $(\mathrm{Mez})$ Arechav. & Mylo & 3 & 0 & 0 & 0 \\
\hline & Myrsine umbellata Mart. & Myum & 3 & 0 & 26 & 5 \\
\hline \multirow{4}{*}{ Rosaceae } & Eriobotrya japonica (Thunb.) Lindl. & Erja & 0 & 0 & 0 & 1 \\
\hline & Prunus myrtifolia (L.) Urb. ${ }^{1}$ & Prmy & 15 & 6 & 10 & 138 \\
\hline & Faramea montevidensis (Cham. \& Schltdl.) DC. & Famo & 3 & 0 & 325 & 17 \\
\hline & Guettarda uruguensis Cham. \& Schltdl. & Guur & 0 & 0 & 2 & 0 \\
\hline \multirow{4}{*}{ Rubiaceae } & Psychotria carthagenensis Jacq. & Psca & 0 & 0 & 141 & 117 \\
\hline & Psychotria leiocarpa Cham. \& Schltdl. & Psle & 0 & 0 & 210 & 60 \\
\hline & Psychotria suterella Müll. Arg. & Pssu & 0 & 0 & 7 & 0 \\
\hline & Rudgea parquioides (Cham.) Müll. Arg. & Rupa & 0 & 0 & 7 & 0 \\
\hline
\end{tabular}


TABELA 1: Continuação...

TABLE 1: Continued...

\begin{tabular}{|c|c|c|c|c|c|c|}
\hline \multirow{2}{*}{ Família } & \multirow{2}{*}{ Espécie } & \multirow{2}{*}{ Rótulo- } & \multicolumn{2}{|c|}{ Estrato superior } & \multicolumn{2}{|c|}{ Estrato inferior } \\
\hline & & & Referência & Plantio & Referência & Plantio \\
\hline \multirow{2}{*}{ Rutaceae } & Zanthoxylum fagara (L.) Sarg. & Zafa & 9 & 1 & 2 & 1 \\
\hline & Zanthoxylum rhoifolium Lam. ${ }^{1}$ & Zarh & 11 & 3 & 6 & 20 \\
\hline \multirow{5}{*}{ Salicaceae } & Banara parviflora (A. Gray) Benth. & Bapa & 1 & 0 & 2 & 0 \\
\hline & Casearia decandra Jacq. & Cade & 2 & 0 & 7 & 0 \\
\hline & Casearia sylvestris $\mathrm{Sw}$. & Casy & 26 & 0 & 9 & 16 \\
\hline & Xylosma tweediana (Clos) Eichler & Xytw & 0 & 0 & 1 & 0 \\
\hline & $\begin{array}{l}\text { Allophylus edulis (A. St.-Hil., A. Juss. \& Cambess.) } \\
\text { Hieron. ex Niederl. }{ }^{1}\end{array}$ & Aled & 34 & 3 & 47 & 36 \\
\hline \multirow[t]{2}{*}{ Sapindaceae } & Cupania vernalis Cambess. & Cuve & 3 & 0 & 40 & 1 \\
\hline & Matayba elaeagnoides Radlk. ${ }^{1}$ & Mael & 2 & 1 & 15 & 3 \\
\hline Sapotaceae & Chrysophyllum marginatum (Hook. \& Arn.) Radlk. & Chma & 7 & 0 & 6 & 5 \\
\hline \multirow{4}{*}{ Solanaceae } & Cestrum intermedium Sendtn. & Cein & 0 & 0 & 5 & 2 \\
\hline & Cestrum strigillatum Ruiz \& Pav. & Cest & 0 & 0 & 1 & 67 \\
\hline & Solanum mauritianum Scop. & Soma & 0 & 0 & 0 & 1 \\
\hline & Solanum pseudocapsicum L. & Sops & 0 & 0 & 0 & 6 \\
\hline Styracaceae & Styrax leprosus Hook. \& Arn. & Stle & 0 & 0 & 4 & 10 \\
\hline Symplocaceae & Symplocos uniflora (Pohl) Benth. ${ }^{1}$ & Syun & 0 & 1 & 0 & 1 \\
\hline \multirow[t]{2}{*}{ Thymelaeaceae } & Daphnopsis racemosa Griseb. & Dara & 0 & 0 & 26 & 39 \\
\hline & Lantana camara $\mathrm{L}$. & Laca & 0 & 0 & 0 & 14 \\
\hline \multirow[t]{2}{*}{ Verbenaceae } & Indeterminada 1 & & 0 & 0 & 0 & 1 \\
\hline & Indeterminada 2 & & 0 & 0 & 1 & 0 \\
\hline
\end{tabular}

(580 ind.ha-1) e quatro mortos, com 28 espécies distribuídas em 18 famílias, sendo Fabaceae aquela com maior riqueza (seis). Dentre os indivíduos vivos desta área, $81 \%$ foram identificados como plantados, sendo que dentre os indivíduos não plantados, a espécie que apresentou maior densidade foi Myrsine coriacea (Sw.) R. Br. Ex Roem. \& Schult. As espécies Schinus terebinthifolius e Psidium clatteyanum se destacaram pelo alto valor de importância (juntas somaram 45,3\%).

A amostragem do estrato inferior da mata ciliar apresentou 2.231 indivíduos distribuídos em 65 espécies e 29 famílias, sendo que as famílias com mais espécies foram Myrtaceae (11) e Rubiaceae (seis). A espécie com maior frequência e densidade foi Mollinedia elegans Tul., seguida de Faramea montevidensis (Cham. \& Schltdl.) DC. Na área em restauração foram encontrados 1.751 indivíduos, pertencentes a 74 espécies e 31 famílias, sendo Myrtaceae (com 13), Fabaceae e Lauraceae (cinco cada) as mais ricas em espécies. Myrsine coriacea apresentou o maior valor de importância (13,0\%), seguida de Leandra australis (Cham.) Cogn. (8,8\%). Em ambas as áreas, predominaram espécies arbóreas (83,1 e 83,8\%, respectivamente para a área de referência e de plantio) em detrimento das arbustivas.

A avaliação da similaridade de Jaccard entre estratos e tratamentos (Tabela 2) indicou que o estrato inferior foi mais similar entre as áreas $(0,56)$ do que qualquer outra comparação, em contraponto com a baixa similaridade entre os estratos superior e inferior do próprio plantio $(0,32)$.

\section{Padrões de estrutura e distribuição espacial da abundância das espécies}

A análise dos descritores estruturais das comunidades evidenciou várias diferenças significativas entre a mata ciliar e a área de plantio, especialmente para o estrato superior no qual todos os parâmetros 
TABELA 2: Valores de similaridade de Jaccard entre os estratos inferior (I) e superior (S), considerando a área de referência de floresta ciliar remanescente e a área de restauração ecológica (plantio), em Cachoeirinha - RS.

TABLE 2: Jaccard's similarity values between the upper and lower strata, whereas the reference of the remaining riparian forest and ecological restoration area (plantation), in Cachoeirinha, RS state.

\begin{tabular}{ccccc}
\hline & Referência - I & Referência - S & Plantio - I & Plantio - S \\
\hline Referência - I & 1,00 & & & \\
Referência - S & 0,51 & 1,00 & & \\
Plantio - I & 0,56 & 0,44 & 1,00 & \\
Plantio - S & 0,22 & 0,32 & 0,32 & 1,00 \\
\hline
\end{tabular}

diferiram (Tabela 3). A área basal total do estrato superior da floresta ciliar remanescente foi cerca de $60 \%$ maior que a observada no plantio, por exemplo. No que diz respeito ao estrato inferior, foi observado que o número de indivíduos, a riqueza e a altura média por unidade amostral não diferem entre os tratamentos.

A análise qualitativa acerca das relações de distribuição das espécies presentes em ambos os estratos das duas áreas (referência $v s$. plantio) pode ser observada no diagrama de ordenação na Figura 1. Nota-se que as unidades amostrais se agrupam dentro de cada tratamento e estrato, o que é corroborado pela análise de MANOVA, a qual indicou diferenças significativas na composição florística entre estratos e entre as áreas $(p<0,001)$. Também é possível observar a maior aproximação entre UA dos estratos inferiores do plantio e da mata ciliar, devido à presença de espécies como Myrsine umbellata Mart. e Daphnopsis racemosa Griseb., por exemplo. As espécies mais relacionadas ao estrato inferior da mata ciliar foram: Mollinedia elegans, Psychotria carthagenensis Jacq., Psychotria leiocarpa Cham. \& Schltdl., Myrcia glabra (O. Berg) D. Legrand e Faramea montevidensis. Já para o estrato inferior da área plantada, as espécies mais relacionadas foram: Myrsine coriacea, Leandra australis e Miconia cinerascens Miq. A presença da espécie Psidium cattleyanum caracterizou a área plantada, tanto no estrato superior quanto no inferior, tendo sido plantada com alta densidade e frequência.

Considerando a composição e abundância de espécies por estrato, as diferenças entre as áreas ficam ainda mais evidentes (Figura 2), sendo que as análises de MANOVA de ambos os estratos indicaram

TABELA 3: Valores médios por unidade amostral dos descritores estruturais e seus respectivos valores de $p$, calculados pela ANOVA comparando a área de referência (floresta ciliar) e a área de restauração ecológica (plantio), em Cachoeirinha - RS.

TABLE 3: Mean values per sampling unit of structural parameters of both the upper and lower stratum of the riparian forest (reference) and restoration planting, in Cachoeirinha, RS state, with indication of $p$ values, obtained by ANOVA.

\begin{tabular}{lcccccc}
\hline \multirow{2}{*}{ Parâmetros } & \multicolumn{3}{c}{ Estrato Superior } & \multicolumn{3}{c}{ Estrato Inferior } \\
\cline { 2 - 7 } & Referência & Plantio & $\mathrm{p}$ & Referência & Plantio & $\mathrm{p}$ \\
\hline Área basal $\left(\mathrm{m}^{2}\right)$ & 0,42 & 0,16 & 0,0001 & - & - & - \\
Abundância (n. indivíduos) & 22,50 & 11,60 & 0,0001 & 111,55 & 87,50 & 0,1182 \\
Riqueza (número de espécies) & 9,55 & 5,25 & 0,0001 & 20,75 & 18,20 & 0,3045 \\
Altura máxima (m) & 10,15 & 6,08 & 0,0002 & 4,67 & 3,19 & 0,0005 \\
Altura média (m) & 6,71 & 4,82 & 0,0001 & 0,95 & 0,83 & 0,1498 \\
Variância da altura & 2,95 & 0,74 & 0,0001 & 0,72 & 0,38 & 0,0134 \\
\hline
\end{tabular}




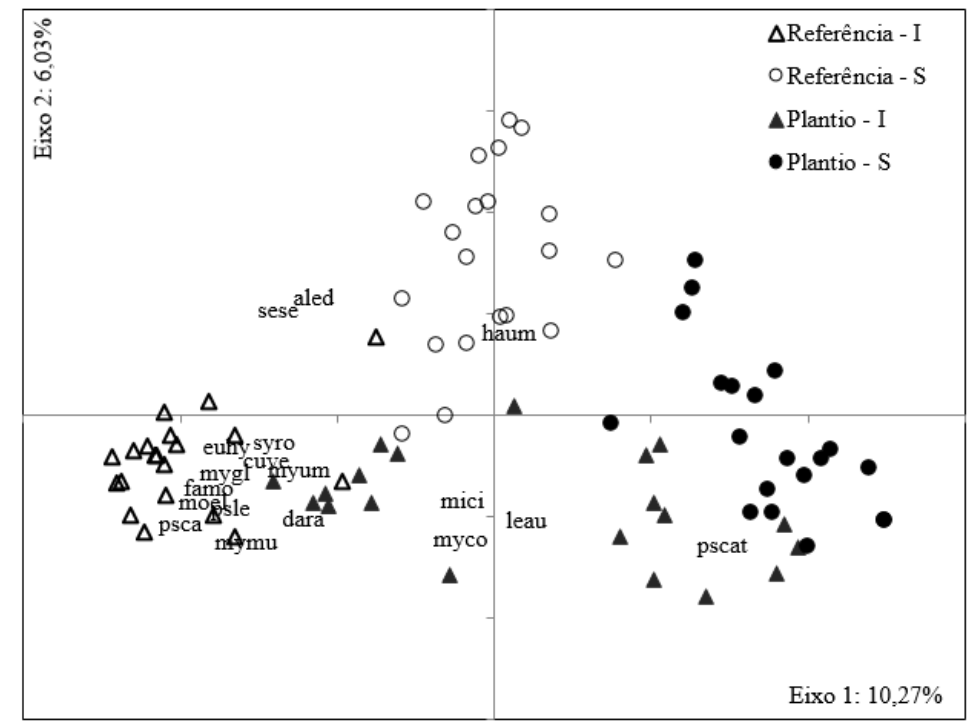

FIGURA 1: Diagrama de dispersão dos dois primeiros eixos da ordenação (PCoA), a partir da similaridade de Jaccard, dos estratos superior (S) e inferior (I) das áreas de referência (floresta ciliar) e restauração (plantio), em Cachoeirinha - RS. As espécies mais correlacionadas aos eixos $(>0,30)$ estão indicadas no diagrama (veja os rótulos na Tabela 1$)$.

FIGURE 1: Scatter diagram of the first two ordination axes (PCoA) based on Jaccard's similarity, considering the upper (S) and lower (I) strata of the reference riparian forest ('reference') and the restoration ('plantio'), in Cachoeirinha, RS state. Species with high correlation to the ordination axes $(>0.30)$ are plotted in the diagram (see labels in Table 1).
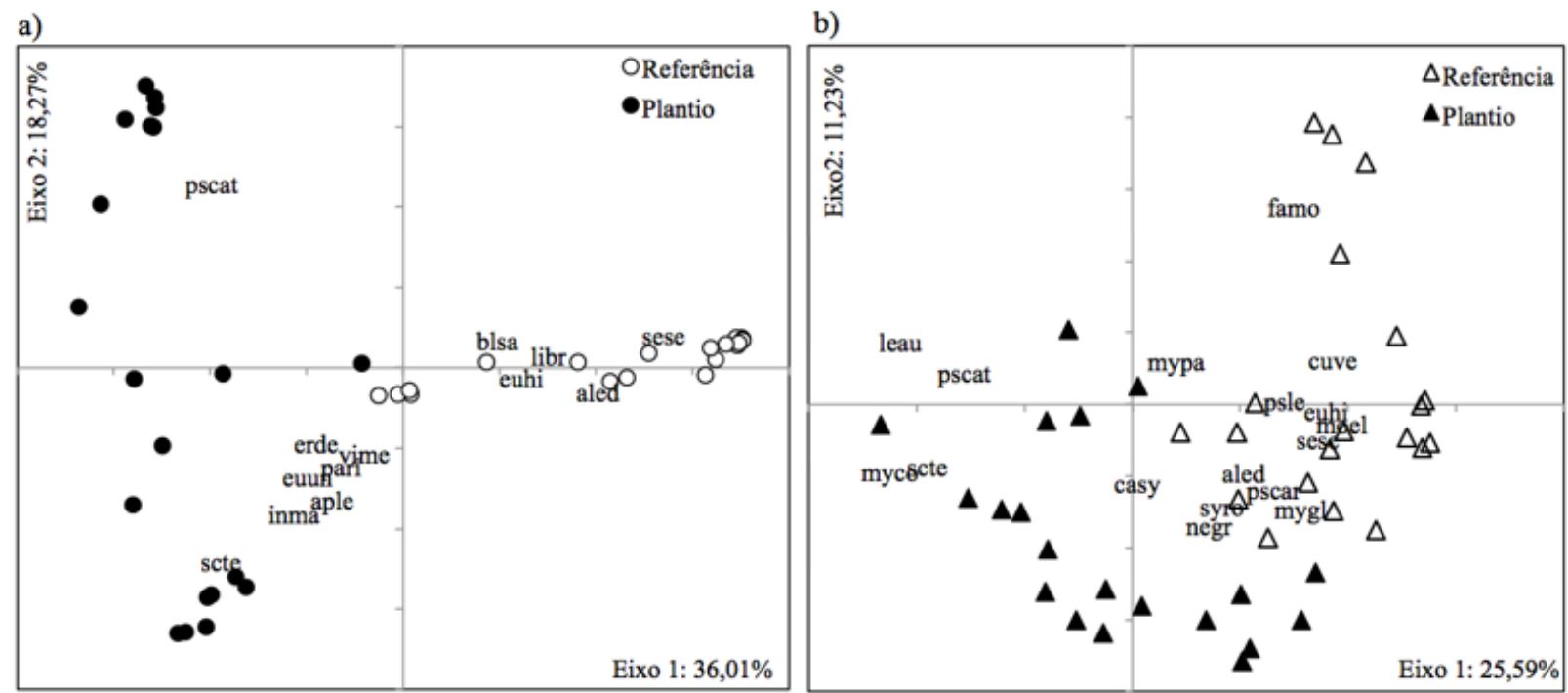

FIGURA 2: Diagrama de dispersão dos dois primeiros eixos da ordenação (PCoA), a partir da distância de Corda entre UA descritas pela abundância de espécies do estrato superior (a) e do estrato inferior (b) nas áreas de referência (floresta ciliar) e restauração (plantio), em Cachoeirinha - RS. As espécies com maior correlação $(>0,30)$ com os eixos estão indicadas nos diagramas (veja os rótulos na Tabela 1).

FIGURE 2: Scatter diagram of the first two ordination axes (PCoA) based on the Cord distance between SU described by species abundance in the upper (a) and the lower (b) stratum of reference riparian forest ('referência') and restoration ('plantio') areas, in Cachoeirinha, RS state. The species most correlated to the axes $(>0.30)$ were indicated in the diagrams (see labels in Table 1). 
diferenças significativas $(p<0,001)$. O diagrama do estrato superior (Figura 2a) demonstra que a composição florística das UA da mata ciliar é mais homogênea (pequena distância entre UA) em termos de espécies dominantes, com destaque a Sebastiania serrata, Lithraea brasiliensis Marchand, Allophylus edulis (A. St.-Hil., A. Juss. \& Cambess.) Hieron. ex Niederl., Luehea divaricata, Handroanthus umbellatus (Sond.) Mattos e Blepharocalix salicifolius (Kunth) O. Berg, pouco frequentes ou ausentes no plantio. Já na área plantada, algumas UA caracterizaram-se pelo predomínio de Psidium cattleyanum e outras por Schinus terebinthifolius, conforme demonstra a variação ao longo do eixo 2. Sob a dominância de S. terebinthifolius também ocorrem com certa densidade as espécies Inga marginata Willd., Apuleia leiocarpa (Vogel) J.F. Macbr., Parapiptadenia rigida (Benth.) Brenan e Vitex megapotamica. Os padrões relacionados ao estrato inferior (Figura $2 b$ ) claramente indicam um estrato espacialmente mais heterogêneo, tanto na floresta ciliar de referência quanto no plantio. A maior variação expressa ao longo do eixo 1 corrobora a diferença florística entre as áreas. Dentre as espécies relacionadas à mata ciliar aparecem Faramea montevidensis, Cupania vernalis Cambess., Eugenia hiemalis Cambess., Mollinedia elegans e Sebastiania serrata, enquanto nas áreas em restauração predominam Myrsine coriacea, Schinus terebinthifolius, Leandra australis e Psidium cattleyanum. Ao mesmo tempo, determinadas espécies ocorrem em ambas as áreas, como, por exemplo, Casearia sylvestris (Sw.), Chrysophyllum marginatum (Hook. \& Arn.) Radlk., Myrcia multiflora (Lam.) DC. e Nectandra grandiflora Nees \& Mart. ex Nees.

A riqueza de espécies regenerantes do estrato inferior apresentou relação linear positiva tanto com a riqueza de espécies plantadas na mesma $\mathrm{UA}$, bem como com o número de indivíduos do estrato superior (Figura 3), sendo ambas significativas $(p<0,005)$.
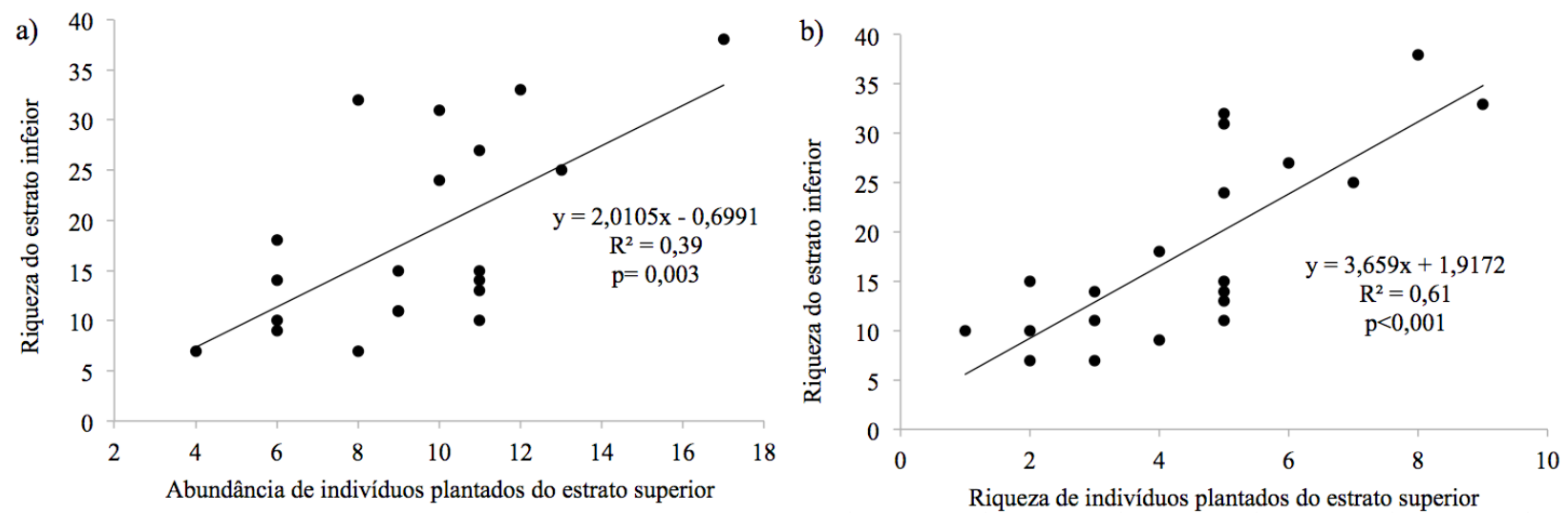

FIGURA 3: Modelos de regressão linear entre a riqueza de espécies do estrato inferior e (a) abundância de indivíduos plantados do estrato superior e (b) riqueza de espécies plantadas do estrato superior, considerando apenas as áreas de restauração, em Cachoeirinha - RS.

FIGURE 3: Linear regression models between the species richness in the lower stratum and (a) the abundance of planted individuals of the upper stratum and (b) the species richness of planted trees, regarding only the restoration areas, in Cachoeirinha, RS state.

\section{DISCUSSÃO}

O recrutamento de plântulas, a diversidade/riqueza, a composição e a estrutura vertical da vegetação são os indicadores mais frequentemente avaliados para definir o sucesso da restauração ecológica (SOCIETY FOR ECOLOGICAL RESTORATION, 2004; RIGUEIRA; MARIANO-NETO, 2013). Os resultados do presente estudo indicam que a regeneração natural está ocorrendo na área que passou por intervenção de restauração ecológica, via plantio de mudas de espécies nativas há cerca de 10 anos. Tanto as espécies que foram, quanto as que não foram plantadas estão regenerando na área, e o padrão de composição e abundância de espécies do estrato inferior indica um processo de sucessão natural com uma similaridade considerável à observada no estrato de regeneração da mata ciliar de referência (Jaccard= 0,56$)$. 
A mata ciliar remanescente, utilizada como referência na comparação dos parâmetros avaliados na área de restauração, apresenta uma composição de espécies e estrutura condizente com as florestas da região (RIO GRANDE DO SUL, 2002; TROIAN et al., 2011). O número de indivíduos por hectare (1.125 ind.ha ${ }^{-1}$ ) pode ser considerado intermediário dentro da amplitude observada para as diferentes florestas do RS (JARENKOW; WAECHTER, 2001), porém, maior que a média indicada para Florestas Estacionais Semideciduais do RS, que é de 929 ind.ha-1 (RIO GRANDE DO SUL, 2002). A riqueza de espécies (51 espécies) encontrada no estrato superior da mata ciliar avaliada neste estudo é considerável. De Marchi e Jarenkow (2008), em um estudo realizado em uma mata ciliar da mesma região fitoecológica, com o mesmo critério de inclusão, porém, maior esforço amostral, encontraram 29 espécies. A riqueza encontrada pode ser reflexo do estado sucessional da floresta, considerado intermediário, uma vez que a faixa de vegetação florestal antes da intervenção por restauração era muito estreita e diversas espécies pioneiras hoje compõem o dossel, como Myrsine coriacea e Trema micrantha (L.) Blume. Mesmo apresentando alta frequência de espécies pioneiras no dossel da floresta, a mesma apresenta dominância de Sebastiania serrata, espécie característica de matas ciliares, que é resistente a ambientes que sofrem inundações e tem preferência por solos úmidos. Além disso, estão presentes outras espécies no estrato superior, tais como Luehea divaricata e Allophylus edulis que são comuns nestas formações (DE MARCHI; JARENKOW, 2008; SOARES; FERRER, 2009).

Dada a estrutura da mata ciliar de referência, era esperada a baixa similaridade com o estrato superior da área de restauração (Jaccard= 0,36 ), mesmo que para o plantio tenham sido utilizadas espécies nativas da região e apesar da proximidade física entre ambas (plantio adjacente ao remanescente). A área de restauração ainda mantém características do plantio, como demonstrado pelas espécies que tiveram maior valor de importância, Schinus terebinthifolius e Psidium cattleyanum, sendo ainda possível identificar as linhas de plantio e traços do coroamento feito por dois anos após o plantio. Por outro lado, os padrões de regeneração expressos pelos dados do estrato inferior demonstram uma clara aproximação entre o plantio e a área de referência. $\mathrm{O}$ estrato inferior por si só apresenta maior heterogeneidade no padrão de distribuição das espécies em ambos os ambientes (maior dispersão das unidades amostrais, Figura 2b). Na mata ciliar, há locais com predomínio de Faramea montevidensis e menor riqueza local, enquanto outros apresentam uma participação de um número maior de espécies, com menor abundância. Nas áreas de plantio, a dominância de uma ou poucas espécies não foi marcante, ocorrendo tanto arbustos de sub-bosque (e.g. Psychotria leiocarpa), quanto arbustos e árvores (e.g. Myrsine coriacea, Sapium glandulosum (L.) Morong, Leandra australis) de espécies tipicamente pioneiras (TABARELLI. MANTOVANI, 1999; PAULA et al., 2004), além de regenerantes de espécies utilizadas nos plantios de restauração (e.g. Psidium cattleyanum, Schinus terebinthifolius). Além disso, várias unidades amostrais se mostraram mais similares à área de referência em termos de composição e densidade de espécies, sendo caracterizadas por regenerantes de espécies arbóreas, como Casearia sylvestris, Chrysophyllum marginatum, Myrcia multiflora e Nectandra grandiflora.

Dentre as espécies encontradas na área plantada, 51 espécies nativas que não foram plantadas estão colonizando a área, vindas provavelmente da mata ciliar adjacente, o que indica um fluxo de dispersão de sementes e um potencial de recuperação da trajetória sucessional das áreas em restauração (SUGANUMA; ASSIS; DURIGAN, 2013), apesar da diferença demonstrada pela análise de variância da composição de espécies. Cabe salientar que tal diferença também ocorreu entre os estratos da própria mata ciliar de referência, o que demonstra a presença de processos dinâmicos de mudança na composição de espécies em uma dada floresta, que por sua vez podem ser resultado de interferência humana passada ou presente (PAULA et al., 2004). No caso do local de estudo, a área passou a ser considerada parque natural a cerca de 10 anos, quando foi retirado o gado e outras possíveis fontes de distúrbio das florestas locais. Assim, ambas as áreas estariam em processo de regeneração natural recente, o que justifica a maior semelhança entre os estratos inferiores e entre o inferior do plantio e o superior da área de referência. Algumas famílias que foram frequentes no levantamento, como Melastomataceae, Rubiaceae e Myrtaceae, são muito importantes na dieta de pássaros frugívoros (CARNEVALE; MONTAGNINI, 2002) e espécies da floresta devem estar se estabelecendo no sub-bosque de ambas as áreas através da dispersão zoocórica. Existe uma forte relação entre a distância dos fragmentos e a intensidade da chuva de sementes, pois quanto maior a distância menor a intensidade de propágulos, principalmente zoocóricos (CUBIÑA; AIDE, 2001; PEREIRA et al., 2013). A área plantada deste estudo está adjacente ao remanescente, ou seja, a distância não seria um limitante, 
mas talvez a presença de determinados animais dispersores sim, tendo em vista o padrão de fragmentação da região (TROIAN et al., 2011).

O tempo necessário à restauração de um sistema florestal depende de inúmeras variáveis locais, regionais e históricas (WORTLEY et al. 2013). Melo e Durigan (2007) colocam que, para a região da Floresta Estacional Semidecidual de São Paulo, 15 anos é a idade aproximada em que os plantios se assemelham estruturalmente (área basal, densidade, altura média e cobertura) à floresta madura. Segundo estes autores, para a riqueza de espécies é necessário um tempo bem mais longo para que se igualem à floresta natural, apesar de ser evidenciado um aumento marcante em riqueza a partir de aproximadamente sete anos, em decorrência dos processos de regeneração natural. No presente estudo, todos as variáveis estruturais analisadas (área basal total, abundância, riqueza, média, máxima e variância da altura dos indivíduos) permaneceram significativamente diferentes entre o estrato superior da mata ciliar de referência e a área de restauração, mesmo após 10 anos do início da intervenção. Aqui salienta-se que na área de plantio, $81 \%$ dos indivíduos amostrados no estrato superior haviam sido plantados e somente o restante teria atingido este estrato via regeneração natural, sendo que alguns, acredita-se que pelo porte, já ocorriam no local. Zanini et al. (2014) indicam que áreas secundárias de floresta pluvial subtropical (norte do RS) atingem padrões estruturais semelhantes a florestas antigas com 25 a 45 anos de sucessão, contudo, a área basal, a diversidade e, principalmente, a composição de espécies permanecem distintos de florestas maduras (ver também LIEBSCH et al., 2007; LIEBSCH et al., 2008; LETCHER; CHAZDON, 2009). Por outro lado, os padrões estruturais observados no estrato inferior evidenciaram certa semelhança à área de referência, inclusive para a riqueza de espécies no nível da unidade amostral, a qual variou consideravelmente entre unidades amostrais (Figura 3b). Em termos de estrutura deste estrato, somente a variância da altura e a altura máxima permaneceram diferentes (menor altura e menor variância - menor complexidade vertical), o que reflete o tempo de regeneração da área (HOLL et al., 2013).

Os resultados deste trabalho evidenciaram ainda uma forte relação linear positiva entre a riqueza observada no estrato inferior da área de restauração e a riqueza e densidade do estrato superior da área plantada. Os dados indicam que as unidades amostrais com baixa riqueza e/ou baixa densidade (menor taxa de pega das mudas) tendem a ter menor riqueza de espécies regenerantes, confirmando a segunda hipótese proposta. Considerando que $81 \%$ dos indivíduos amostrados neste estrato foram plantados, é possível inferir que decisões de manejo e tipo de intervenção na restauração florestal (como tempo de tratos culturais das mudas, número e distribuição espacial das espécies plantadas) terão influência significativa na capacidade de regeneração natural. De acordo com Carnevale e Montagnini (2002), a riqueza de espécies da regeneração natural é maior em áreas em que um conjunto diverso de espécies é utilizado nos plantios, pois proporcionam nichos variados para a fauna dispersora e, portanto, um conjunto maior de espécies tem potencial de se estabelecer. Da mesma forma, como a densidade também foi importante, presume-se que a maior proporção de sombreamento e poleiros também auxilia, fornecendo condições para o estabelecimento de um número maior de espécies nas áreas de plantio.

\section{CONCLUSÕES}

O presente estudo demonstrou que áreas de restauração ecológica com plantio de mudas nativas, adjacentes à mata ciliar remanescente, demonstram capacidade de regeneração natural em um curto período pós-intervenção (10 anos). Estas áreas apresentaram uma estrutura do estrato inferior parcialmente semelhante à observada no sub-bosque da floresta de referência, inclusive em termos de riqueza de espécies regenerantes por unidade amostral, porém, a composição florística ainda permaneceu distinta. Isto se deve, em parte, ao fato de a regeneração das próprias espécies utilizadas no plantio, as quais, apesar de serem nativas da região, são pouco frequentes em matas ciliares e foram plantadas em densidades elevadas (e.g. Psidium cattleyanum, Schinus terebinthifolius). A escolha das espécies utilizadas nos plantios é fator essencial para recompor matas ciliares com menor dissimilaridade, facilitando o fluxo entre áreas restauradas e remanescentes em prol da regeneração natural dos ecossistemas restaurados.

Sabe-se que a escolha das espécies para plantios de restauração ecológica florestal tem barrado na dificuldade em se encontrar um conjunto diversificado de mudas de espécies nativas e características do sistema em foco. No caso de florestas ciliares, quando o objetivo da restauração é o retorno da área a uma 
trajetória sucessional similar à referência, é importante considerar espécies ribeirinhas, como as do gênero Sebastiania que apresentam elevados valores de importância nas matas ciliares do RS, mas não são (ou muito raramente) utilizadas em plantios de restauração pela falta de disponibilidade em viveiros.

Por fim, como a riqueza e a densidade de espécies plantadas diretamente afetaram a riqueza das espécies em regeneração natural, este estudo reforça a importância dos plantios com uma certa diversidade de espécies nativas para o processo de retomada da trajetória sucessional em áreas de restauração florestal.

\section{AGRADECIMENTOS}

Agradecemos a todos os colegas que ajudaram na elaboração deste trabalho, em especial a Rodrigo S. Bergamin e Martin Molz pela identificação de algumas espécies. Ao Instituto de Biociências da UFRGS e ao LEVEG pelo apoio logístico, ao CNPq pela bolsa à última autora (Processo: 307719/2012-0). À empresa Souza Cruz por autorizar o trabalho no Parque e à Sandra B. M. Fontanella pela gentil recepção no Parque e auxílio nas saídas de campo.

\section{REFERÊNCIAS}

ANGIOSPERM PHYLOGENY GROUP III. An update of the Angiosperm Phylogeny Group classification for the orders and families of flowering plants: APG III. Botanical Journal of the Linnean Society, London, v. 161, n. 2, p. 105-121, 2009.

CARNEVALE, N. J.; MONTAGNINI, F. Facilitating regeneration of secundary forests with the use of mixed and pure plantations of indigenous tree species. Forest Ecology and Management, Netherlands, v. 163, p. 217-227, 2002.

CUBIÑA, A.; AIDE, T. M. The effect of distance from forest edge on seed rain and soil seed banck in a tropical pasture. Biotropica, Gainesville, v. 33, p. 260-267, 2001.

DE MARCHI, T. C.; JARENKOW, J. A. Estrutura do componente arbóreo de mata ribeirinha no rio Camaquã, município de Cristal, Rio Grande do Sul, Brasil. Iheringia, Série Botânica, Porto Alegre, v. 63, n. 2, p. 241-248, 2008.

GUARIGUATA, M. R.; OSTERTAG, R. Neotropical secundary forest succession: changes in structural and functional characteristics. Forest Ecology and Management, Netherlands, v. 148, n. 1, p. 185-206, 2001. HOLL, K. D. Restoring Tropical Forest. Nature Education Knowledge, Ithaca, v. 4, n. 4, p. 4, 2013.

IBGE. Manual técnico da vegetação brasileira. 2. ed. Brasília: IBGE, 2002. (Manuais técnicos em geociências, 1).

IBGE. Mapa da vegetação do Brasil e Mapa de biomas do Brasil. Brasília: IBGE; Ministério do Meio Ambiente, 2004.

JARENKOW, J. A.; WAECHTER, J. L. Composição, estrutura e relações florísticas do componente arbóreo de uma floresta estacional no Rio Grande do Sul, Brasil. Revista Brasileira de Botânica, São Paulo, v. 24, n. 3, p. 263-272, 2001.

KAUANO, E. E. et al. Micro- and meso-scale factors affect the restoration of Atlantic Forest. Natureza \& Conservação, Rio de Janeiro, v. 11, n. 2, p. 145-151, 2013.

LEGENDRE, P.; LEGENDRE, L. Numerical ecology. 3. ed. Amsterdam: Elsevier, 2012. 990 p.

LEITE, P. F. Contribuição ao conhecimento fitoecológico do sul do Brasil. Revista Ciência \& Ambiente, Santa Maria, n. 24, p. 51-73, 2002.

LETCHER S. G.; CHAZDON, R. L. Rapid recovery of biomass, species richness, and species composition in a forest chronosequence in northeastern Costa Rica. Biotropica, Gainesville, v. 41, n. 5, p. 608-617, 2009.

LIEBSCH, D.; GOLDENBERG, R.; MARQUES, M. C. M. Florística e estrutura de comunidades vegetais em uma cronossequência de Floresta Atlântica no Paraná. Acta botanica Brasilica, Belo Horizonte, v. 21, p. 983-992, 2007.

LIEBSCH, D.; MARQUES, M. C. M.; GOLDENBERG, R. How long does the Atlantic Rain Forest take to recover after a disturbance? Changes in species composition and ecological features during secondary succession. Biological Conservation, Boston, v. 141, p. 1717-1725, 2008.

MANLY B. F. J. Randomization and Monte Carlo Methods in Biology. London: Chapman \& Hall, 1991. $281 \mathrm{p}$. 
MELO, A. C. G.; DURIGAN, G. Evolução estrutural de reflorestamentos de restauração de matas ciliares no Médio Vale do Paranapanema, SP - Brasil. Scientia forestalis, Piracicaba, v. 101, n. 73, p. 101-111, 2007.

MORENO, J. A. Clima do Rio Grande do Sul. Porto Alegre: Governo do Estado do Rio Grande do Sul Secretaria da Agricultura, 1961. 41 p.

MORO, M. F.; MARTINS, F. R. Métodos de levantamento do componente arbóreo-arbustivo. In: FELFILI, J. M. et al. (eds.). Fitossociologia no Brasil. [s. 1.: s. n.], 2011. v. 1, p. 174-212.

NERY, E. R. A. et al. O conceito de restauração na literatura científica e na legislação brasileira. Revista Caititu, Salvador, n. 1, p. 43-56, 2013.

OVERBECK, G. E. et al. Restoration Ecology in Brazil - Time to step out of the Forest. Natureza \& Conservação, Rio de Janeiro, v. 11, n.1, p. 92-95, 2013.

PAULA, A. et al. Sucessão ecológica da vegetação arbórea em uma Floresta Estacional Semidecidual, Viçosa, MG, Brasil. Acta Botânica Brasileira, Belo Horizonte, v. 18, n. 3, p. 407-423, 2004.

PEREIRA, L. C. S. M.; OLIVEIRA, C. C. C.; TOREZAN, J. M. D. Woody species regeneration in Atlantic Forest restoration sites depends on surrounding landscape. Natureza \& Conservação, Rio de Janeiro, v. 11, n. 2, p. 138-144, 2013.

PILLAR V. D. How accurate and powerful are randomization tests in multivariate analysis of variance? Community Ecology, Budapest, n. 14, p. 153-163, 2013.

PIOVESAN, J. C. et al. Processos ecológicos e a escala da paisagem como diretrizes para projetos de restauração ecológica. Revista Caititu, Salvador, v. 1, n. 1, p. 57-72, 2013.

RIGUEIRA, D. M. G.; MARIANO-NETO, E. Monitoramento: uma proposta integrada par avaliação do sucesso em projetos de restauração ecológica em áreas florestais brasileiras. Revista Caititu, Salvador, n. 1, p. 73-88, 2013.

RIO GRANDE DO SUL. Secretaria Estadual do Meio Ambiente. Inventário Florestal Contínuo do Rio Grande do Sul. Porto Alegre: FATEC/SEMA, 2002. Disponível em: <http://w3.ufsm.br/ifcrs/frame.htm>. Acesso em: 24 nov. 2013.

RODRIGUES, R. R.; LEITÃO FILHO, H. F. Matas ciliares: conservação e recuperação. 2. ed. São Paulo: Editora da Universidade de São Paulo; Fapesp, 2001. 320 p.

SOCIETY FOR ECOLOGICAL RESTORATION. Grupo de Trabalho sobre Ciência e Política. 2004. Princípios da SER International sobre a restauração ecológica. Disponível em: <http://www.ser.org/ docs/default-document-library/ser-primer-portuguese.pdf $>$. Acesso em: 1 ago. 2013.

SOARES, L. R.; FERRER, R. S. Estrutura do componente arbóreo em uma área de floresta ribeirinha na bacia do rio Piratini, Rio Grande do Sul, Brasil. Biotemas, Florianópolis, v. 22, n. 3, p. 47-55, 2009.

SOBRAL, M. et al. Flora arbórea e arborescente do Rio Grande do Sul, Brasil. São Carlos: RiMa, 2006. $350 \mathrm{p}$.

STANTURF, J. A.; PALIK, B. J.; DUMROESE, R. K. Contemporary forest restoration: a review emphasizing function. Forest Ecology and Management, Netherlands, v. 331, p. 292-323, 2014.

SUGANUMA, M. S.; ASSIS, G. B.; DURIGAN, G. Changes in plant species composition and functional traits along the successional trajectory of a restored patch of Atlantic Forest. Community Ecology, Budapest, v. 15, n. 1, p. 27-36, 2014.

TABARELLI, M.; MANTOVANI, W. A regeneração de uma floresta tropical montana após corte e queima (São Paulo-Brasil). Revista Brasileira de Biologia, São Carlos, v. 59, n. 2, p. 239-250, 1999.

TEIXEIRA, M. B. (Coord.). Plano ambiental de Cachoeirinha. Porto Alegre: MCT, 2007. v. 2. Disponível em: <http://www.cachoeirinha.rs.gov.br/portal/index.php/plano-ambiental-municipal $>$. Acesso em: 1 ago. 2013.

TRES, D. R. et al. Poleiros Artificiais e Transposição de Solo para a Restauração Nucleadora em Áreas Ciliares. Revista Brasileira de Biociências, Porto Alegre, v. 5, p. 312-314, 2007.

TROIAN, L. C. et al. Florística e padrões estruturais de um fragmento florestal urbano, região metropolitana de Porto Alegre, RS, Brasil. Iheringia, Série Botânica, Porto Alegre, v. 66, n. 1, p. 5-16, jul. 2011.

WORTLEY, L.; HERO, J. M.; HOWES, M. Evaluating Ecological restoration success: a review of the literature. Restoration Ecology, Hoboken, v. 21, n. 5, p. 537-543, set. 2013.

ZANINI, K. J. et al. Atlantic rain forest recovery: successional drivers of floristic and structural patterns of secondary forest in Southern Brazil. Journal of Vegetation Science, Edinburgh, v. 25, n. 4, p. 1056-1068, 2014. 